\title{
The Lamb Shift Effect (on Series Limits) Revisited
}

\author{
Peter F. Lang \\ Birkbeck College, University of London, London, UK \\ *Corresponding Author: Peter F. Lang, Birkbeck College, University of London, London, UK

\begin{abstract}
This work briefly introduces the development of atomic spectra and the measurement of the Lamb shift. The importance of series limits/ionization energies is highlighted. The use of specialist computer programs to calculate the Lamb shift and ionization energies of one and two electron atoms are mentioned. Lamb shift values calculated by complex programs are compared with results from an alternative simple expression. The importance of experimental measurement is discussed. The conclusion is that there is a case to experimentally measure series limits and re-examine the interpretation and calculations of the Lamb shift.
\end{abstract}

Keywords: Atomic spectra, series limit, Lamb shift, ionization energies, relativistic corrections.

\section{INTRODUCTION}

The study of atomic spectra is an integral part of physical chemistry. Measurement and examination of spectra dates back to the nineteenth century. It began with the examination of spectra which stemmed from the observation that the spectra of hydrogen consist of a collection of lines and not a continuous emission or absorption.

It was found by examination of various spectra that each chemical element produced light only at specific places in the spectrum. First attempts to discover laws governing the positions of spectral lines were influenced by the view that vibrations which give rise to spectral lines might be similar to those in sound and might correspond to harmonical overtones of a single fundamental vibration. The early studies led to many important discoveries. There are numerous important publications related to atomic spectra, series limits/ionization energies or the lamb shift. It is not possible to list all of them and we are only able to refer to a small selection of these papers in the discussion below.

The spectrum of hydrogen was naturally the most studied. Stoney (1871) found that the first, second and fourth lines of the hydrogen spectrum were inverse ratios of the numbers 20, 27, and 32. Huggins (1880) discovered in the spectra of some stars a number of prominent lines which seemed to be a continuation of the regular series of the hydrogen lines in the visible spectrum. Balmer attempted to discover an equation which could express the wavelengths of various lines of the spectra satisfactorily. He eventually arrived at an empirical expression which related four lines of the now famous "Balmer" (1885) series as members of a converging series of lines. Lyman (1914), Paschen (1908), Brackett (1922), and Pfund (1924), all made contributions to the inverstigations of the spectrum of hydrogen. The next important development in the measurement of spectra was made by Rydberg (1890) who developed a simple law on emission spectra of elements when he proposed that "in the spectra of the elements there are series of rays whose wavelengths are functions of consecutive integral numbers".

As knowledge of the spectrum of hydrogen increased, more complex spectra such as those of the alkaline earths were also studies. However, even though experimental spectroscopy developed rapidly in the late nineteenth century, there was little understanding of the origin of atomic spectra. Theoretical interpretations of spectra began at the beginning of the twentieth century. Firstly, Einstein (1906) pointed out the importance of Planck's theory on the mathematical relationship between intensity and wavelength that describes the spectrum of a glowing object at a given temperature. Planck's (1910) historic contribution was his statement that the energy of an oscillator is not continuously variable, but is restricted to integral multiples of the frequency of the oscillator and a constant, which is now named after its discoverer. 
Bohr (1913), who developed the theory of the constitution of atoms, brought together the German school of theoretical physics and the English school of experimental physics and laid the foundations of quantum theory. Besides the development of quantum theory, the study of atomic is of great importance in astrophysics. The measurement and analysis of atomic spectra enabled astrophysicists to understand a tremendous amount about cosmology, the constitution and life of stars, the solar system and other aspects of astronomy (Snow, 1991). In his original theory, Bohr used the rest mass of the electron in his calculations of the energies of lines. With the development of the theory of relativity it was obvious that at high velocities, the electron orbits (in his theory) were not circular and relativistic change of mass need to be considered. Not long afterwards Sommerfeld made a detailed study of non radiating non-circular orbits. By applying all the known theories including relativistic corrections Sommerfeld formulated his well known fine structure formula and produced general rules of atomic spectra and atomic structure. All that was discussed in great detail in his book (Sommerfeld, 1934). Calculated results on energy levels of hydrogen like atoms using Sommerfeld's rules agreed well with experimental results known at the time. The next major step in the understanding of atomic structure and spectra came from Schrödinger (1926), who introduced a mathematical technique competent to deal with the wave particle duality of matter and energy. Schrödinger's contribution and amongst others, Heisenberg's (1930) work gave rise to a more complex form of quantum theory. Schrödinger's wave equation is not relativistic. In a series of papers, Dirac $(1925,1927,1928)$ was able to make a general formulation of quantum mechanics on a firm footing. He produced a relativistic equation which when applied to the hydrogen atomic allow rigorous solution for the energy levels. According to Dirac theory the ${ }^{2} \mathrm{~S}_{1 / 2}$ and ${ }^{2} \mathrm{P}_{1 / 2}$ level of the $\mathrm{H}_{\alpha}$ line should be of the same level. However, the large line width of the line which was yet unresolved prevented a definite check between theory and experiment. As more observations were made, there was suspicion that there may be a splitting of the line not accounted for by theory. Work in the nineteen thirties to detect the absorption of energy from high frequency spark gap oscillators by excited atoms in a hydrogen discharge did not yield definitive results. However, the development of microwave technology in the early nineteen forties allowed Lamb and Retherford to study the possibility of stimulating transitions between fine structure levels of the same principal quantum number $n$. Their experiment depends on the long life time of the ${ }^{2} S_{1 / 2}$ energy level. Lamb and Retherford $(1947,1950,1951,1952)$ put forward a scheme to make a beam of atomic hydrogen and to excite it by electron bombardment. Most of the atoms in the $2^{2} \mathrm{~S}_{1 / 2}$ level, but not those in the $2^{2} \mathrm{P}_{1 / 2}$ level would have a sufficiently long life time to be detected. In their experiments, hydrogen is introduced into an evacuated atomic beam chamber through a tungsten oven heated to $2500^{\circ} \mathrm{K}$ where the molecules dissociate. Cross bombardment of the beam by electrons excites the atoms and deviates them. The excited atoms then pass into the radiofrequency interaction space, between the inner and outer conductors of a transmission line, which they enter through slots in the outer cylinder. They are detected by the ejection of electrons from a tungsten plate on which they fall. The current constituted by the ejected electrons is amplified and measured. Their work showed that there is an upward shift of the components of $2^{2} \mathrm{~S}_{1 / 2}$ level by about $1000 \mathrm{M}$ hertz (equivalent to just over $0.03 \mathrm{~cm}^{-1}$ ). Full details of their experiments were published in a series of papers in the Physical Review. Lamb et al (1953) later made refinements to the apparatus and the repeated experiments produced similar results but with more precision. The detailed experiments of Lamb, Retherford and their team demonstrated that the fine structure of the second quantum level of hydrogen does not agree with the Dirac theory of the hydrogen atom. Bethe (1947) was amongst the first to attempt to produce a method to calculate the Lamb shift which led to the development of quantum electrodynamics. The new theory recognizes that the mass of the electron is finite, and that this finite quantity must embrace the apparently infinite self-energy. By the method now commonly called "re-normalization of mass", Bethe was able to eliminate the effect of the infinite electromagnetic mass of a point electron and obtained a calculated Lamb shift of 1040 mega Hertz which was in very good agreement with the experimental value produced by Lamb and Retherford.

The experiments of Lamb and Retherford confirming the existence of the Lamb shift generated much excitement and interests in the spectrum of atom hydrogen. Many researchers performed experiments using different methods to measure the Lamb shift, initially of hydrogen (Newton et al 1969, Van Wijngaarden et al 1974, Lundeen et al 1981). Gradually interest in the Lamb shift measurements of other atoms also became apparent (Leventhal et al 1972, Drake et al 1979, Zacek et al 1984, Eikema et al 1997). Mean while, there is still much interest in the measurements for the hydrogen spectrum ( 
Weitz et al 1994, Bourzeix et al 1996, Van Wijngaarden 1998). In terms of the calculation of the Lamb shift, there is great interest and a whole host of publications are available discussing the merits of the different methods (Baraneer et al 1953, Seke 1994, Cavicchi et al 1996, Evans et al 2011). The aim of this paper is to stress the importance of experimental measurements (or the lack of) of ionization energies and Lamb shifts (which influence the series limits) for one and two electron atomic ions from the first member of the series all the way to the very heavy elements and stimulate discussion on what the Lamb shift represents.

\section{METHOD}

For a particular atom or ion the separation between the spectral lines is constant and usually measured in wave numbers (reciprocal wave lengths or $\mathrm{cm}^{-1}$ ). Each line in a spectrum represents an electron moving from one energy state to another energy state or from one atomic orbital to another atomic orbital. Usually, a series of discrete lines can be observed to get closer and closer to each other and reaches a limit which is followed by a continuous spectrum (or continuum). The start of the continuum which is the end of the series, or series limit, corresponds to the separation or capture of an electron with zero velocity. If the transition takes place from the ground state to the ionised state, the wave number of the series limit gives the ionisation energy of the electron (Herzberg 1944).

Studies of atomic spectra and series limits are essential to determining the electronic configurations of elements and ionization pathways (Lang and Smith 2003, 2010). Since the series limit of an atomic spectrum shows the ionization energy, such information is useful in establishing the electronegativity value of an element (Lang and Smith 2015) and understanding thermodynamic aspects of many chemical reactions (Lang and Smith 2014). This wave number is often converted to and presented in electron volts $(\mathrm{eV})$.

A considerable number of the measurements of atomic spectra individual elements are reported in the Journal of the Optical Society of America. There are also many spectra reported in other journals such as Arkiv för Fysik. Besides papers on individual spectra, there are a whole host of compilations of spectra and some are published in the Journal of Physical and Chemical Reference Data. The most comprehensive compilation of atomic spectra are contained in three volumes containing wavenumbers and atomic energy levels preceded the critical survey by Moore $(1949,1952,1958)$ of ionization limits for atoms and atomic ions. Ionization energies derived from spectra and calculations ranging from crude approximations to highly complex equations based on quantum mechanical theory are accompanied by assessments of reliability. These three volumes were followed by a further volume containing ionization limits derived from optical spectra (Moore 1970). Many recent papers on spectra and ionization limits refer back to Moore's work. An extensive volume by Martin, Zalubus, and Hagan (1978) reviewed energy levels and ionization limits for rare earth elements. The CRC Handbook of Chemistry and Physics also contains authoritative data of ionization energies from the above publications and later sources. Besides experimental measurements, there are also many theoretical calculations of spectra limits/ ionization energies of atoms and atomic ions or Lamb shift topics reported in the journals (Hylleraas and Midtdal 1956, Pekeris 1958, Drake et al 1999).

The precision of the measured values of series limits/ionization energies vary greatly between papers. Some spectral lines quoted, may consist of lines with wavelengths "tentatively identified" or "predicted" or "smoothed along isoelectronic sequence" or "calculated" (Shirai et al 2000). Authors sometimes do not provide conversion factors or values of fundamental constants used and that make the results less precise. Uncertainties of series limits can range from a few wave numbers or less (less than $0.001 \mathrm{eV}$ ) to over $10000 \mathrm{~cm}^{-1}$ (over $1 \mathrm{eV}$ ) Lang and Smith 2016). When there is insufficient experimental measurements and the series limit/ionization energy is estimated/calculated using an extrapolation formula and the degree of uncertainty/errors are not provided and the ionization energy is given to the nearest 1000 or 10000 or $100000 \mathrm{~cm}^{-1}$ (and when converted to $\mathrm{eV}$ no experimental error values are provided). The values provided by all compilations including the publications by Moore for the one electron series are all calculated and not experimental values.

Since the success of Bethe's calculation of the Lamb shift value of atomic hydrogen, the general trend in theoretical calculations is to use vastly sophisticated arrays of complex equations and specialist computer programs to solve those equations. Garcia and Mack (1965) were the first to publish values of energy levels and ionizatiion energies of one electron atoms, calculated by a computer, whose accuracy "exceeds that of currently achievable experimental values". Their computed values included 
the Dirac energy, a correction due to nonseparability of the Dirac equation in terms of reduced masses, a correction for the finite size of the nuclear charge distribution plus a correction for the finite nuclear mass and a quantum electrodynamical correction. Soon after, Mdtdal and Aashamar (1967) reported values of two electron atoms using perturbation theory. They made use of perturbation theory expansions of the energy corrections and covered a correction due to the variation of mass with velocity, a correction to the interaction between the two electrons originating from the retardation of the electromagnetic field produced by a moving charge, the spin-orbit interaction, correction originating in a term characteristic of the Dirac theory, interaction between the spins of the two electrons, mass polarization and the Lamb shift correction. Since these two early papers other theoretical energy levels and ionization energies or Lamb shift values for one and two electron atomic systems calculated by specialist computer software have been published. For example, in 1977 Erickson (1977) reported series limits/ionization energies of one electron atoms with values slightly different from those computed by Garcia and Mack. Mohr (1983) computed Lamb shifts of some energy levels of one electron atoms. Mohr's work took account of mass corrections, self-energy correction, vacuum polarization, nuclear size effect, relativistic recoil correction and higher order radiative corrections. This was soon followed by Johnson and Soff (1985) who published computed values of the Lamb shift and ionization energies of hydrogen-like atoms. Then came a paper by Drake (1988) on theoretical energies for the helium isoelectronic sequence. More recent work on energy levels and ionization energies(Sansonetti 2006) still refers back to the earlier papers such as values computed by Drake and Erickson.

In the Dirac theory, for the spectrum of atomic hydrogen atom, there should be no splitting between the $2 s_{1 / 2}$ and $2 p_{1 / 2}$ levels since they have the same energy. However, the experiments of Lamb and Retherford showed otherwise and that the $2 s_{1 / 2}$ level is slightly higher in energy than the $2 p_{1 / 2}$ level. The shift in energy for the hydrogen atom when $n=1$ (the ground state) is very small and cannot be measured directly. Experimentally measured ionization energies (series limits), if they are available, compared to values calculated by the relativistic Dirac equation and with results computed by quantum electrodynamic equations (see references for Garcia and Mack, Mdtdal and Aashamar, Johnson and Soff, Drake and Erickson) can determine the true amount of energy shift in one electron atomic ions attributed to the "Lamb shift".

Lang and Smith (1013) developed an alternative relativistic equation which did not require the use of specialist computer software. Ionization energies including the Lamb shift can be calculated from this simple equation using a hand held calculator and values produced by this equation showed exceptionally very good agreement with ionization energy values provided by Garcia and Mack (1965), Johnson and Soff (1985), Erikson (1977) and Drake (1988). In the proposed relativistic equation, the ionization energy of a one electron atom is simply:

$\mathrm{I}=\mu\left(1 / 2 \mathrm{~m}_{\mathrm{o}} \mathrm{v}^{2}+0.5\left(1 / 4 \mathrm{~m}_{\mathrm{o}} \mathrm{v}^{4} / \mathrm{c}^{2}+3 / 16 \mathrm{~m}_{\mathrm{o}} \mathrm{v}^{6} / \mathrm{c}^{4}\right)-\mathrm{E}_{\mathrm{l}}\right)$

The Lamb Shift $\left(\mathrm{E}_{\mathrm{l}}\right)$ is usually interpreted as a quantum electrodynamic effect and is calculated by complex computer programs. In the relativistic equation the Lamb shift is considered to be an effect caused by a combination of mass and size and motion of whole system. Since the ground state is not a state of zero motion of two particles without dimensions but a state of two finite sized particles in motion there should be a reduction in the overall relativistic correction. The energy reduction is approximately equal to the following expression:

$E_{1}=m_{e} /\left(m_{e}+m_{p}\right)\left(\alpha / 2^{.667}\right)\left(1 / 2 m_{o} v_{o}^{2}\right)(Z-S)^{3.2} A^{1 / 3}$

where $m_{e}$ is the electron mass, $m_{p}$ is the proton mass, where the factor for the reduced mass correction for hydrogen is $\mathrm{m}_{\mathrm{e}} /\left(\mathrm{m}_{\mathrm{e}}+\mathrm{m}_{\mathrm{p}}\right) . \alpha$ is the fine structure constant and $(\alpha / 2.667)$ is an approximation of the square root of the ratio of nuclear to atomic size for hydrogen. A is the mass number of the atom. $\mathrm{S}$ is the screening constant and in a one electron system $\mathrm{S}$ is zero. The size of the nucleus increases roughly in proportion to $\mathrm{A}^{1 / 3}$.

The ionization energy of a two electron system is $\left(E_{k}-E_{1}-E_{s} \pm E_{r}\right)$ where:

$$
\begin{aligned}
& \mathrm{E}_{\mathrm{k}}=\mu\left(1 / 2 \mathrm{~m}_{\mathrm{o}} \mathrm{v}^{2}+0.45\left(1 / 4 \mathrm{~m}_{\mathrm{o}} \mathrm{v}^{4} / \mathrm{c}^{2}+3 / 16 \mathrm{~m}_{\mathrm{o}} \mathrm{v}^{6} / \mathrm{c}^{4}\right)\right) \\
& \mathrm{E}_{\mathrm{l}}=\mu\left(\mathrm{m}_{\mathrm{e}} /\left(\mathrm{m}_{\mathrm{e}}+\mathrm{m}_{\mathrm{p}}\right)\left(\alpha / 2^{2 / 3}\right)\left(1 / 2 \mathrm{~m}_{\mathrm{o}} \mathrm{v}_{\mathrm{o}}^{2}\right)(\mathrm{Z}-0.5)^{3.2} \mathrm{~A}^{1 / 3}\right) \\
& \mathrm{E}_{\mathrm{s}}=0.25 \mu\left(1 / 2 \mathrm{~m}_{\mathrm{o}}\left(\mathrm{v}_{\mathrm{o}}(\mathrm{Z})\right)^{2}-1 / 2 \mathrm{~m}_{\mathrm{o}}\left(\mathrm{v}_{\mathrm{o}}(\mathrm{Z}-0.5)\right)^{2}\right) \\
& \mathrm{E}_{\mathrm{l}}=\left(-\left(1 / 2 \mathrm{~m}_{\mathrm{o}} \mathrm{v}_{\mathrm{o}} 2\right) \mathrm{QI} \sqrt{ }\left(\left(\mathrm{r}_{\mathrm{e}} / \mathrm{r}_{\mathrm{B}}\right) /(\mathrm{Z}) !\right)\right)
\end{aligned}
$$


$r_{e}$, the electron radius, is $\mathrm{e}^{2} / 4 \pi \varepsilon_{0} \mathrm{~m}_{\mathrm{e}} \mathrm{c}^{2}$ and $\mathrm{r}_{\mathrm{B}}$ is the Bohr radius $\left(\varepsilon_{0} \mathrm{~h}^{2} / \pi \mathrm{m}_{\mathrm{e}} \mathrm{c}^{2}\right)$.

Full details [63] of the derivation of the relativistic equation are described in previous work and not discussed here.

\section{RESULTS}

Table 1 shows a comparison of the calculated ionization energies (series limits) in wave numbers ( $\mathrm{cm}^{-}$

${ }^{1}$ ) for one electron ions (given to eight or nine figures depending on the published data). All the values shown are in very good agreement and biggest difference between any two sets is 20 ppm (parts per million) or no greater than $0.002 \%$.

Table1. Comparison of theoretical series limits for the one electron isoelectronic sequence $\left(\mathrm{cm}^{-1}\right)$.

\begin{tabular}{|l|l|l|l|l|}
\hline Atom & Lang \& Smith & Garcia \& Mack & Erickson & Johnson \& Soff \\
\hline $\mathrm{H}$ & 109678.773 & 109678.764 & 109678.774 & 109678.771 \\
\hline $\mathrm{He}$ & 438908.480 & 438908.850 & 438908.885 & 438908.877 \\
\hline $\mathrm{Li}$ & 987659.345 & 987660.945 & 987661.027 & 987661.008 \\
\hline $\mathrm{Be}$ & 1756016.21 & 1756018.67 & 1756018.82 & 1756018.79 \\
\hline $\mathrm{B}$ & 2744104.91 & 2744107.6 & 2744107.87 & 2744107.90 \\
\hline $\mathrm{C}$ & 3952064.60 & 3952061.20 & 3952061.52 & 3952061.67 \\
\hline $\mathrm{N}$ & 5380097.5 & 5380089 & 5380089.3 & 5380089.8 \\
\hline $\mathrm{O}$ & 7028409.7 & 7028393 & 7028393.6 & 7028394.7 \\
\hline $\mathrm{F}$ & 8897253 & 8897240 & 8897240.3 & 8897242.5 \\
\hline $\mathrm{Ne}$ & 10986917 & 10986875 & 10986873.1 & 10986877.2 \\
\hline $\mathrm{Na}$ & 13297712 & 13297676 & 13297672 & 13297680 \\
\hline $\mathrm{Mg}$ & 15830030 & 15829946 & 15829938 & 15829950 \\
\hline $\mathrm{Al}$ & 18584214 & 18584138 & 18584123 & 18584143 \\
\hline $\mathrm{Si}$ & 21560776 & 21560628 & 21560601 & 21560631 \\
\hline $\mathrm{P}$ & 24760079 & 24759942 & 24759900 & 24759942 \\
\hline $\mathrm{S}$ & 28182776 & 28182834 & 28182460 & 28182526 \\
\hline $\mathrm{Cl}$ & 31829236 & 31829065 & 31828900 & 31828983 \\
\hline $\mathrm{Ar}$ & 35699986 & 35699936 & 35699780 & 35699895 \\
\hline $\mathrm{K}$ & 39796238 & 39795863 & 39795630 & 39795784 \\
\hline $\mathrm{Ca}$ & 44118128 & 44117545 & 44117210 & 44117409 \\
\hline
\end{tabular}

Garcia and Mack and Erickson did not tabulate the Lamb shift values in their results and only Johnson and Soff listed the relevant Lamb shift values computed for one electron ions. Table 2 shows the Lamb shift component of the series limits calculated by Lang and Smith and computed by Johnson and Soff (given to five figures).

Table2. Values of the Lamb shift for one electron ion $s\left(\mathrm{~cm}^{-1}\right)$

\begin{tabular}{|l|l|l|}
\hline Atom & Lang \& Smith & Johnson \& Soff \\
\hline $\mathrm{H}$ & .27458 & .27262 \\
\hline $\mathrm{He}$ & 4.0055 & 3.5926 \\
\hline $\mathrm{Li}$ & 17.667 & 15.956 \\
\hline $\mathrm{Be}$ & 48.233 & 45.507 \\
\hline $\mathrm{B}$ & 105.32 & 101.93 \\
\hline $\mathrm{C}$ & 194.31 & 196.20 \\
\hline $\mathrm{N}$ & 334.99 & 340.20 \\
\hline $\mathrm{O}$ & 536.95 & 546.70 \\
\hline $\mathrm{F}$ & 828.90 & 829.10 \\
\hline $\mathrm{Ne}$ & 1181.3 & 1201.3 \\
\hline $\mathrm{Na}$ & 1678.9 & 1677.0 \\
\hline $\mathrm{Mg}$ & 2249.7 & 2272.0 \\
\hline $\mathrm{Al}$ & 3022.8 & 3000.0 \\
\hline $\mathrm{Si}$ & 3878.5 & 3878.0 \\
\hline $\mathrm{P}$ & 5003.6 & 4921.0 \\
\hline $\mathrm{S}$ & 6216.9 & 6146.0 \\
\hline $\mathrm{Cl}$ & 7776.8 & 7568.0 \\
\hline $\mathrm{Ar}$ & 9762.6 & 9206.0 \\
\hline $\mathrm{K}$ & 11509 & 11069 \\
\hline $\mathrm{Ca}$ & 13676 & 13184 \\
\hline
\end{tabular}


Table 3 is a comparison of the calculated ionization energies (series limits) in wave numbers $\left(\mathrm{cm}^{-1}\right)$ for two electron ions (given to nine figures with the exception of the hydride ion). All the values shown are in good agreement and biggest difference between any two sets is still less than $100 \mathrm{ppm}$ (parts per million). Mdtdal and Aashamar as well as Drake tabulated the Lamb shift values computed for two electron ions. The Lamb shift values are shown in Table 4.

Table3. Calculated series limits for two electron ions $\left(\mathrm{cm}^{-1}\right)$

\begin{tabular}{|l|l|l|l|}
\hline Atom & Lang \& Smith & Midtdal \& Aashamar & Drake \\
\hline $\mathrm{H}$ & 6054.546 & 6083.132 & \\
\hline $\mathrm{He}$ & 198310.525 & 198310.758 & 198310.655 \\
\hline $\mathrm{Li}$ & 610080.745 & 610079.644 & 610078.526 \\
\hline $\mathrm{Be}$ & 1241330.41 & 1241259.43 & 1241256.60 \\
\hline $\mathrm{B}$ & 2092154.89 & 2092003.23 & 2091995.45 \\
\hline $\mathrm{C}$ & 3162689.96 & 3162440.71 & 3162423.35 \\
\hline $\mathrm{N}$ & 4453121.67 & 4452757.64 & 4452723.34 \\
\hline $\mathrm{O}$ & 5963633.49 & 5963134.62 & 5963073.04 \\
\hline $\mathrm{F}$ & 7694442.08 & 7693134.62 & 7693706.59 \\
\hline $\mathrm{Ne}$ & 9645791.26 & 9645002.91 & 9644840.74 \\
\hline $\mathrm{Na}$ & 11817947.3 & 11817060.7 & 11816816.8 \\
\hline $\mathrm{Mg}$ & 14211241.5 & 14210260.6 & 14209907.8 \\
\hline $\mathrm{Al}$ & 16825967.2 & 16825021.4 & 16824528.9 \\
\hline $\mathrm{Si}$ & 19662564.9 & 19661692.0 & 19661022.7 \\
\hline $\mathrm{P}$ & 22721344.5 & 22720765.0 & 22719877.9 \\
\hline $\mathrm{S}$ & 2600871.8 & 26002661.5 & 26001512.9 \\
\hline $\mathrm{Cl}$ & 29507461.1 & 29507948.2 & 29506490.3 \\
\hline $\mathrm{Ar}$ & 33235563.9 & 33237170.4 & 33235354.5 \\
\hline $\mathrm{K}$ & 37188256.1 & 37190815.5 & 37188598.2 \\
\hline $\mathrm{Ca}$ & 41365636.3 & 41369605.1 & 41366937.5 \\
\hline
\end{tabular}

Table4. Lamb shift values for two electron ions (to two decimal places)

\begin{tabular}{|l|l|l|l|}
\hline Atom & Lang \& Smith & Midtdal \& Aashamar & Drake* \\
\hline $\mathrm{He}$ & 1.59 & 1.27 & 1.38 \\
\hline $\mathrm{Li}$ & 9.84 & 7.83 & 8.94 \\
\hline $\mathrm{Be}$ & 31.39 & 27.11 & 30.00 \\
\hline $\mathrm{B}$ & 75.01 & 65.70 & 73.64 \\
\hline $\mathrm{C}$ & 146.75 & 132.24 & 150.28 \\
\hline $\mathrm{N}$ & 203.66 & 235.22 & 271.40 \\
\hline $\mathrm{O}$ & 435.76 & 383.04 & 449.31 \\
\hline $\mathrm{F}$ & 688.77 & 583.68 & 697.00 \\
\hline $\mathrm{Ne}$ & 1000.17 & 844.46 & 1028.08 \\
\hline $\mathrm{Na}$ & 1443.43 & 1171.87 & 1456.62 \\
\hline $\mathrm{Mg}$ & 1958.78 & 1571.31 & 1997.05 \\
\hline $\mathrm{Al}$ & 2660.20 & 2046.99 & 2664.15 \\
\hline $\mathrm{Si}$ & 3444.57 & 2601.71 & 3473.11 \\
\hline $\mathrm{P}$ & 4478.98 & 3236.78 & 4439.18 \\
\hline $\mathrm{S}$ & 5603.50 & 3951.82 & 5578.07 \\
\hline $\mathrm{Cl}$ & 7052.11 & 4744.70 & 6905.43 \\
\hline $\mathrm{Ar}$ & 8900.67 & 5611.40 & 8437.17 \\
\hline $\mathrm{K}$ & 10543.52 & 6545.85 & 10189.88 \\
\hline $\mathrm{Ca}$ & 12583.80 & 7539.94 & 12179.44 \\
\hline
\end{tabular}

In Drake's paper, a value of the quantum electrodynamic effect is given for each ion and is considered as same as the Lamb shift in this work.

\section{DISCUSSION}

There are two practical reasons for attempting to gain a better understanding of the Lamb shift. Firstly, it enables physical scientists to appreciate how theory is developing and how close theory can represent the real world since all theories are only approximate models of reality. Secondly, better understanding of the Lamb shift leads to better understanding of atomic spectra and atomic spectra is 
an essential tool in indentifying the elements present in stars and their relative abundance. This in turn, lead to a better understanding of the universe. The above comparisons in Tables 1 to 4 show on the one hand that, excluding the Lang and Smith equation, even though the ionization energies/series limits of one and two electron ions are calculated using highly complex theory and computed by special computer programs they are not in complete agreement and the values produced by the simple Lang and Smith equation in most cases agree well with those computed by complex formulas. For ionization energies/series limits of three to ten electrons some of the published values are accurately measured and many, especially the higher members of each isoelectronic sequence, are calculated using extrapolation formulas (Brittin and Odabasi 1971). Lang and Smith have shown that their relativistic equation calculated values of ionization energies of three to five electron ions which agree very well the values published in the CRC Handbook (2011-2012) (which are also calculated values). A very simple non relativistic equation developed by Lang and Smith (2013) can be used to calculate ionization energies of five or more electron isoelectronic atomic ions and are shown to give values which agree well with those compiled in the CRC Handbook.

Leventhal (1973) measured the Lamb shift of heavy ions and concluded that measured Lamb shift values of heavy ions were not good examples to test critically the theoretical values of the Lamb shift. Lundeen and Pipkin (1981) measured the Lamb shift in hydrogen and commented that it was not in good agreement with theory. Pellegrin et al (1982) measured the Lamb shift of hydrogenic phosphorus and obtained a value of $20.18 \mathrm{~T} \mathrm{~Hz}$, whilst Pross et al (1993) obtained an experimental Lamb shift measurement of $18.839 \mathrm{~T} \mathrm{~Hz}$ for hydrogenic phosphorus as opposed to a theoretical value of $20.254 \mathrm{~T} \mathrm{~Hz}$. Nakamura et al (2003)- obtained an experimental value of $28.7 \mathrm{eV}$ for $\mathrm{Rh}^{44+}$ as opposed to a theoretical value of $25.13 \mathrm{eV}$. However, if the experimental uncertainty of $3.4 \mathrm{eV}$ is included, then experimental and theoretical values agree. The theoretical computations of the ionization energies/series limits and Lamb shifts for one and two electron atomic ions are getting more and more complex and readers of published papers are not possible to examine or appreciate the level of detail included in the computer programs that compute the required values.

Lang and Smith did not take account of mass polarization or nuclear size effects for both one and two electron ions. Johnson and Soff considered the self energy correction, nuclear size effect on self energy correction, higher order vacuum polarization correction, shift due to the Uehling potential, nuclear size effect on the Uehling potential correction, higher order radiative correction, nuclear size correction to the Dirac energy, relativistic recoil correction, relativistic reduce mass correction all to be contributions to the Lamb shift. Midtdal \& Aashamar considered mass polarization as separate from the Lamb shift and Drake excluded both mass polarization and nuclear size correction from the Lamb shift (quantum electrodynamic effects) correction. Drake also discussed the agreement between experiment and theory for the energy shift which results in the splitting between $2 s_{1 / 2}$ and $2 p_{1 / 2}$ with the $2 s_{1 / 2}$ level slightly higher in energy. He showed that for many atoms the agreement is good but for a few atoms there is significant discrepancy between theory and experiment and the difference can be as much as 1 electron volt (equivalent to $8065 \mathrm{~cm}^{-1}$ ). Bethe called the Lamb shift the "electromagnetic shift" of energy levels. Hence, it may be most appropriate that all corrections of energy shifts due to an electromagnetic or relativistic effect not accounted for by the Dirac theory should be included as components of the "Lamb shift".

\section{CONCLUSION}

Atomic spectra and series limits/ionization energies are an important part of physical science and the current situation regarding series limits, ionization energies and measurements of Lamb shifts is not completely satisfactory. The above Tables and discussion show that it is useful to measure values of ionization energies of one and two electron atomic ions. Experimental results can then be compared with theory and theory or theoretical computations may have to be modified and/or improved if computed results do not agree well with experiment. Secondly there appear to be different interpretations of the term "Lamb shift". It needs to be clearly defined and universally agreed. There is also a case to evaluate what corrections should be included in the Lamb shift (or quantum electrodynamic effect).

\section{ACKNOWLEDGEMENT}

This paper is dedicated to the late Dr. Barry C. Smith who had made an important contribution to the interpretation of trends in ionization energies. 


\section{REFERENCES}

Balmer, J. J. (1885), Notiz uber die Spektrallinien des Wasserstoffs, Ann. Der Phys.,261, 80-87.

Baraneer, M., Bethe, H. A., Feynman, R. P.(1953), Relativistic corrections to the Lamb shift, Phys. Rev., 92, 482-502.

Bethe, H. A.(1947), The electromagnetic shift of energy levels, Phys. Rev., 72, 339-344.

Bohr, N. (1913), On the constitution of atoms and molecules, Phil. Mag., 26, 476-502.

Bourzeix., S., de Beavoir, B., Nez. F., Pluminer, M. D., de Tomasi, F., Julien, L., Biraben, F. (1996), High resolution spectroscopy of the hydrogen atom - determination of the $1 \mathrm{~S}$ Lamb shift, Phys. Rev. Lett., 76, 384-387.

Brackett, F. S. (1922), Visible and Infrared radiation of hydrogen, Astrophys J. 1922, 154-158.

Brittin, W.E., Odabasi, H. (ed.) (1971), Topics in modern physics - a tribute to E. U. Condon, Adam Hilger, London, UK, 133-134.

Cavicchi, M., Vairo, A.(1996), A new tool for the Lamb shift calculation, Zeit. Phys. C, 63, 455-462.

Dirac, P. A. M. (1925), The fundamental equations of quantum mechanics, Proc. Roy. Soc., 25, 642-655.

Dirac, P. A. M. (1927), The quantum theory of the emission and absorption of radiation, Proc. Roy. Soc.,114, 243-265.

Dirac, P. A. M. (1928), The quantum theory of the electron, Proc. Roy. Soc., 117, 610-624.

Drake, G. W. F., Goldman, S. P., Van Wijngaarden, A. (1979), $\mathrm{He}^{+}$lamb shift measurement by the quenching radiation anisotropy method, Phys. Rev., A, 20, 1299-1306.

Drake, G. W. F., Goldman, S. P.(1999), Bethe logarithms for $\mathrm{Ps}^{-}, \mathrm{H}^{-}$and heliumlike atoms, Can. J. Phys., 77 , 835-845.

Drake, G. W.(1988), Theoretical energies for the $\mathrm{n}=1$ and 2 states of the helium isoelectronic sequence up to $\mathrm{Z}=100$, Can. J. Phys., 66, 586-611.

Eikema, K. S. E., Ubachs, W., Vassen., W., Hogervoost, H. (1997), Lamb shift measurement in the 1s ground state of helium, Phys. Rev. A, 55, 1866-1885.

Einstein, A. (1906), Uber einen die Erzeugung und Verwandlung des Lichtes betreffenden heuristischen Gesichtspunkt, Ann. der Phys.,322, 132-149.

Erickson, G. W.(1977), Energy levels of one electron atoms, J. Phys. Chem. Ref. Data, 6, 831-851.

Evans, M. W., Eckart, H.(2011), The Lamb shift in atomic hydrogen calculated from Einstein-Cartan-Evans (ECE) field theory, J. Found. Phys. Chem., 1, 493-520.

Pfund, A. H. (1924), The emission of nitrogen and hydrogen in infrared, J. Opt. Soc. Am., 9, 193-196.

Garcia, J. D., Mack, J. E. (1965), Energy level and line tables for one-electron atomic spectra, J. Opt. Soc. Am., 55, 654-685.

Haynes, W. M.(ed) (2011-2012), CRC Handbook of Chemistry and Physics, $92^{\text {nd }}$ edn., CRC, Boca Raton, RL, USA, 10:196-10:198.

Heisenberg, W. (1930), The physical principles of the quantum theory, Dover, UK.

Herzberg, G.(1944), Atomic spectra and atomic structure, Dover, New York, USA, p.15.

Huggins W.(1880), On the Photographic Spectra of Stars, Phil. Trans, 171, 669-690.

Hylleraas, E A., Midtdal, J.(1956), Ground state energy of two-electron atoms, Phys. Rev., 103, 829-530.

Johnson, W. R., Soff, G. (1985), The Lamb shift in hydrogen-like atoms $1 \leq Z \leq 110$, Atm. Data Nucl. Data Tab., 33, 405-445.

Lamb, W. E., Retherford, R. C. (1947), Fine structure of the hydrogen atom by a microwave method, Phys. Rev.,72,

241-247.

Lamb, W. E., Retherford, R. C. (1950), Fine structure of the hydrogen atom , Part I, Phys. Rev., 79, 349372.

Lamb, W. E., Retherford, R. C (1951), Fine structure of the hydrogen atom, Part II, Phys. Rev., 81, 222-232.

Lamb, W. E., Retherford, R. C. (1952), Fine structure of the hydrogen atom, Part III, Phys. Rev., 85, 259-276.

Lamb, W. E., Retherford, R. C. (1952), Fine structure of the hydrogen atom, Part IV, Phys. Rev., 86, 10141022.

Lang, P. F., Smith, B. C.(2003), Ionization energies of atoms and atomic ions, J. Chem. Edu.,80, 938-946.

Lang, P. F., Smith, B. C. (2010), Ionization energies of lanthanides, J. Chem. Edu., 87, 875-881.

Lang, P. F., Smith, B. C. (2015), An equation to calculate internuclear distances of covalent, ionic and metallic lattices, Phys. Chem. Chem. Phys., 17, 3355-3369. 
Lang, P. F., Smith, B. C.(2014), Thermodynamic aspects of the chemistry of copper, silver and gold. Aust. J. Ed. Chem., 34-38.

Lang, P.F., Smith, B.C. (2016), Measurement and Calculation of Ionization Energies of Atoms and Atomic Ions, J. Basic \& Applied Res. Int., 15, 301-308.

Lang, P. F., Smith, B. C. (2013), Medthods of calculating ionization energies of multielectron (five or more) isoelectronic atomic ions, Sci. World. J., DOI: 10.1155/2013/157412.

Lang, P. F., Smith, B. C. (2013), Relativistic corrections for calculating ionization energies of one to five electron isoelectronic atomic ions, ISRN Inorg. Chem., Vol. 2013, article ID 689040, 10 pages.

Leventhal, M., Murnick, D. E., Kugel, H. W. (1972), Lamb shift measurement for the $n=2$ state of hydrogenic ${ }^{16}$ O., Phys. Rev. Lett., 28, 1609-1611.

Leventhal, M. (1973), Lamb shift measurements in heavy ions, Nucl. Instr. Meth., 110, 343-353.

Lundeen, S. R., Pipkin, F. M.(1981), Measurement of the Lamb shift in hydrogen n=2, Phys. Rev. Lett., 46, 232-236.

Lyman, T. (1914), An extension of the spectrum in the extreme ultra violet, Nature, 93, 241-245.

Martin, W. C., Zalubas, R., Hagan, L.(1978), Atomic Energy Levels - the Rare Earth Elements, NSRDS-NBS 60, US Department of Commerce, Washington DC., USA.

Midtdal, J., Aashamar, K. (1967), Perturbation theory expansions through 20th order of mass polarization correction, relativistic effects and Lamb shift of the two-electron system $(1 \mathrm{~s})^{2}{ }^{1} \mathrm{~S}$, Phys. Norvegica, 2, 100108.

Mohr, P. J.(1983), energy levels of hydrogen-like atoms predicted by quantum electrodynamics $10 \leq Z \leq 40$, Atm. Dat. Nucl. Dat.Tab., 29, 453-466.

Moore, C. E. (1949), Atomic energy levels Volume 1, US Department of Commerce, Washington, DC., USA.

Moore, C. E. (1952), Atomic energy levels Volume 2, US Department of Commerce, Washington, DC., USA.

Moore, C. E.(1958), Atomic energy levels Volume 3, US Department of Commerce, Washington, DC., USA.

Moore, C. E.(1970), Ionization potentials and ionization limits derived from the analysis of optical spectra. NSRDS-NBS 34, US Department of Commerce, Washington, DC., USA.

Nakamura, N., Nakahara, T., Ohtani, S. (2003), The 1S Lamb shift in hydrogen like rhodium measured with an electron beam ion trap, J. Phys. Soc. Japn., 72, 1650-1654.

Newton, G., Andrews, A., Unsworth, P. J. (1969), A precision determination of the Lamb shift in hydrogen, Phil. Trans. Roy. Soc., 290, 373-404.

Paschen, F. (1908), Zur Kenntnis ultraroter Linienspektra I, Ann. der Phys., 332, 537-570.

Pekeris. C. L.1958), Ground state of two-electron atoms, Phys. Rev., 112, 1649-1659.

Pellegrin, P., El Masri, Y., Palffy, L., Prieels, R. (1982), Lamb shift measurement in hydrogenic phosphorus, Phys. Rev. Lett, 49, 1762-1764.

Planck, M. (1910), Zur Theorie der Warmestrahlung, Ann. der Phys., 336, 758-768.

Pross, H., Budelsky, D., Kremer, L., Platte, D., von Brenthano, P., Gassen, J., Mueller, D., Scheuer, F., Pape, A., Sens, J.C.(1993), Lamb shift measurement in hydrogenic phosphorus, Phys. Rev. A, 25, 1875-1890.

Rydberg, J. R. (1890), On the structure of the line spectra of the chemical elements, Phil. Mag., 331-337.

Sansonetti, J. E.(2006), Wavelength, transition probabilities, and energy levels for the spectra of rubidium $\left(\mathrm{Rb}_{\mathrm{I}}\right.$ through $\mathrm{Rb}_{\mathrm{Xxx} \text { III }}$, J. Phys. Chem. Ref. Data, 35, 301-417.

Schrödinger, E. (1926), Uber des Verhaltnis der Heisenberg-Born-Jordanschen Quantenmechanik zu der meinem, Ann. der Phys., 384, 734-756.

Seke, J.(1994), Calculation of the discrete spectrum Lamb shift contribution in atomic hydrogen, Physica A, 203, 284-297.

Shirai, T., Sugar, J., Musgrove, A., Wiese, W. L.(2000), Spectral data for highly ionized atoms Ti, V, Cr, Mn, $\mathrm{Fe}, \mathrm{Co}, \mathrm{Ni}, \mathrm{Cu}, \mathrm{Kr}$, Mo, J. Phys. Chem. Ref. Data, Monograph No. 8.

Snow., T. P. (1991), The Dynamic Universe, $4^{\text {th }}$ ed., West Publishing, St. Paul, U.S.A., pp. 110-121, 159-162, 389-396, 414-423, 559-565, 630-632

Sommerfeld, A. (1934), Atomic structure and spectral lines, Methuen, London, UK.

Stoney, G. J.(1871), On the cause of the interrupted spectra of gases, Phil. Mag., 41, 291-296. Triebwasser, S., Dayhoff, E. S., Lamb, W. E. (1953), Fine structure of the hydrogen atom, Part V, Phys. Rev., 89, 98-105.

Triebwasser, S., Dayhoff, E. S., Lamb, W. E. (1953), Fine structure of the hydrogen atom, Part VI, Phys. Rev., 89, 106-111. 
Van Wijngaarden, A., Drake, G. W. F., Farago, P.S. (1974), A new method for Lamb shift measurement, Phys. Rev. Lett., 33, 4-7.

Van Wijngaarden, A., Holuj, F., Drake, G. W. F. (1998), Lamb shift measurement in hydrogen by the anisotropy method, Can. J. Phys., 76, 95-103.

Weitz, M., Huber, A., Schmidt Kaler, F., Leibfried, D., Hansch, T. W. (1994), Precision measurement of the hydrogen and deuterium 1S ground state Lamb shift, Phys. Rev. Lett., 72, 328-331.

Zacek, V., Bohn, H., Brum, H., Faestermann, T., Von Feilitzsch, F., Georginis, G., Kienle, P., Schuhbeck, S. (1984), Lamb shift measurement in hydrogen like sulphur, Zeit. Phys. A, 318, 7-11.

Citation: P. Lang, "The Lamb Shift Effect (on Series Limits) Revisited", International Journal of Advanced Research in Chemical Science (IJARCS), vol. 4, no. 11, pp. 10-19, 2017. http://dx.doi.org/10.20431/23490403.0411002

Copyright: () 2017 Authors. This is an open-access article distributed under the terms of the Creative Commons Attribution License, which permits unrestricted use, distribution, and reproduction in any medium, provided the original author and source are credited. 